\title{
The Structure of $\mathrm{CCl}_{5}{ }^{-}$in the Gas Phase
}

\author{
Timothy R. Corkish, Christian T. Haakansson, Allan J. McKinley, Duncan A. Wild
}

School of Molecular Sciences, The University of Western Australia, Crawley, Western Australia, 6009, Australia

\section{SUPPORTING INFORMATION}

The supporting information presented here comprises results of ab initio calculations performed on $\mathrm{Cl}^{-} \ldots \mathrm{CCl}_{4}$ gas phase anion complexes, and the $\mathrm{CCl}_{5}{ }^{-}$transition state. Included are the energies, vibrational data, and cartesian coordinates predicted at the MP2 and CCSD(T) levels of theory, with aug-cc-pVTZ, QZ, and 5Z basis sets. The aug-cc-pV(X+d)Z basis sets were used for chlorine. Collectively, these basis sets will be referred to as AVXZ. 


\section{Energetics}

Table S1: Energies of the bare chloride anion and chlorine radical determined from $\operatorname{CCSD}(\mathrm{T})$ calculations.

\begin{tabular}{|c|c|c|c|c|c|c|c|}
\hline & & $\begin{array}{c}\mathrm{E}_{(\mathrm{CCSD}(\mathrm{T}))} \\
{\left[E_{h}\right]} \\
\end{array}$ & $\begin{array}{l}\text { VDE } \\
{[\mathrm{eV}]} \\
\end{array}$ & $\begin{array}{c}\text { Experimental SO* } \\
{[\mathrm{eV}]}\end{array}$ & $\begin{array}{l}\text { Split } \\
{[\mathrm{eV}]}\end{array}$ & $\begin{array}{c}\text { Lit. }{ }^{2} P_{3 / 2}{ }^{\dagger} \\
{[\mathrm{eV}]}\end{array}$ & $\begin{array}{c}\text { Shift } \ddagger \\
{[\mathrm{eV}]}\end{array}$ \\
\hline \multirow[t]{4}{*}{$\mathrm{Cl}^{-} \mid \mathrm{Cl}$} & AVTZ & $-459.8066265 \mid-459.6778578$ & 3.504 & & $3.468 \mid 3.577$ & & +0.145 \\
\hline & AVQZ & $-459.6958913 \mid-459.8283951$ & 3.606 & & $3.570 \mid 3.679$ & & +0.043 \\
\hline & AV5Z & $-459.7013126 \mid-459.8348346$ & 3.633 & & $3.597 \mid 3.706$ & & +0.016 \\
\hline & CBS & $-459.7066880 \mid-459.8412328$ & 3.661 & $-0.036 \mid+0.073$ & $3.625 \mid 3.734$ & 3.613 & -0.012 \\
\hline
\end{tabular}

* Values from http://www.nist.gov/pml/data/handbook/index.cfm

$\dagger$ Values from http://webbook.nist.gov

$\ddagger$ Shift refers to the difference between the predicted and literature Electron Detachment Energy

Table S2: Energies of the $\mathrm{C}_{3 \mathrm{v}}$ halogen-appended $\mathrm{Cl}^{-} \ldots \mathrm{CCl}_{4}$ gas phase anion complex and neutral counterpart predicted from $\operatorname{CCSD}(\mathrm{T})$ calculations.

\begin{tabular}{|c|c|c|c|c|c|c|c|}
\hline & & $\begin{array}{c}\operatorname{Anion}_{(\mathrm{CCSD}(\mathrm{T}))} \\
{\left[E_{h}\right]}\end{array}$ & $\begin{array}{c}\operatorname{Neutral}_{(\mathrm{CCSD}(\mathrm{T}))} \\
{\left[E_{h}\right]}\end{array}$ & $\begin{array}{c}\text { zpe } \\
{\left[\mathrm{kJ} \mathrm{mol}^{-1}\right]}\end{array}$ & $\begin{array}{c}\mathrm{D} e \\
{\left[\mathrm{~kJ} \mathrm{~mol}^{-1}\right]}\end{array}$ & $\begin{array}{c}\mathrm{D}_{0}^{\dagger} \\
{\left[\mathrm{kJ} \mathrm{mol}^{-1}\right]}\end{array}$ & $\begin{array}{l}\mathrm{VDE}^{\dagger} \\
{[\mathrm{eV}]}\end{array}$ \\
\hline \multirow[t]{4}{*}{$\mathrm{Cl}^{-} \ldots \mathrm{CCl}_{4}$} & AVTZ & -2336.7998247 & -2336.6479978 & & & & \\
\hline & AVQZ & -2336.9106662 & -2336.7549268 & $26.7^{*}$ & & & \\
\hline & AV5Z & -2336.9447580 & -2336.7879148 & & & & \\
\hline & CBS & -2336.9785121 & -2336.8205701 & & 47.5 & 46.7 & $4.23 \mid 4.34$ \\
\hline
\end{tabular}

* MP2/AVQZ value

$\dagger$ Determined using CCSD(T)/CBS energies and MP2/AVQZ zpe

Table S3: Energies of the $\mathrm{C}_{3 \mathrm{v}} \mathrm{Cl}^{-} \ldots \mathrm{CCl}_{4}$ gas phase anion complex, featuring the halide at the face of the $\mathrm{CCl}_{4}$ molecule, and neutral counterpart predicted from $\operatorname{CCSD}(\mathrm{T})$ calculations.

\begin{tabular}{|c|c|c|c|c|c|c|c|}
\hline & & $\begin{array}{c}\operatorname{Anion}_{(\operatorname{CCSD}(\mathrm{T}))} \\
{\left[E_{h}\right]}\end{array}$ & $\begin{array}{c}\operatorname{Neutral}_{(\operatorname{CCSD}(\mathrm{T}))} \\
{\left[E_{h}\right]}\end{array}$ & $\begin{array}{c}\text { zpe } \\
{\left[\mathrm{kJ} \mathrm{mol}^{-1}\right]}\end{array}$ & $\begin{array}{c}\mathrm{D} e \\
{\left[\mathrm{~kJ} \mathrm{~mol}^{-1}\right]}\end{array}$ & $\begin{array}{c}\mathrm{D}_{0}^{\dagger} \\
{\left[\mathrm{kJ} \mathrm{mol}^{-1}\right]}\end{array}$ & $\begin{array}{l}\mathrm{VDE}^{\dagger} \\
{[\mathrm{eV}]}\end{array}$ \\
\hline \multirow[t]{4}{*}{$\mathrm{Cl}^{-} \ldots \mathrm{CCl}_{4}$} & AVTZ & -2336.7899093 & -2336.6539300 & & & & \\
\hline & AVQZ & -2336.9006398 & -2336.7607232 & $26.6^{*}$ & & & \\
\hline & AV5Z & -2336.9347036 & -2336.7937173 & & & & \\
\hline & CBS & -2336.9684218 & -2336.8296220 & & 21.0 & 20.2 & $3.72 \mid 3.83$ \\
\hline
\end{tabular}

* MP2/AVQZ value

$\dagger$ Determined using CCSD(T)/CBS energies and MP2/AVQZ zpe 


\section{Vibrational Data}

Table S4: Vibrational frequencies for the halogen-appended $\mathrm{Cl}^{-} \ldots \mathrm{CCl}_{4}$ gas phase anion complex from MP2/AVQZ calculations. Frequencies in $\mathrm{cm}^{-1}$, zero point energies (zpe) in $\mathrm{kJ} \mathrm{mol}^{-1}$, force constants in $\mathrm{mDyne} \AA^{-1}$.

\begin{tabular}{cccc}
\hline Mode & Symmetry & Frequency & Force constant \\
\hline$\omega_{1}$ & $\mathrm{~A}_{1}$ & 786 & $4.749^{*}$ \\
$\omega_{2}$ & $\mathrm{E}$ & 775 & 4.609 \\
$\omega_{3}$ & $\mathrm{E}$ & 775 & 4.609 \\
$\omega_{4}$ & $\mathrm{~A}_{1}$ & 448 & 4.128 \\
$\omega_{5}$ & $\mathrm{E}$ & 326 & 2.048 \\
$\omega_{6}$ & $\mathrm{E}$ & 326 & 2.048 \\
$\omega_{7}$ & $\mathrm{~A}_{1}$ & 309 & 1.799 \\
$\omega_{8}$ & $\mathrm{E}$ & 226 & 1.053 \\
$\omega_{9}$ & $\mathrm{E}$ & 226 & 1.053 \\
$\omega_{10}$ & $\mathrm{~A}_{1}$ & 125 & $0.317 *$ \\
$\omega_{11}$ & $\mathrm{E}$ & 68 & 0.093 \\
$\omega_{12}$ & $\mathrm{E}$ & 68 & 0.093 \\
\hline zpe & 26.7 & & \\
\hline
\end{tabular}

*Respective $\mathrm{Cl}-\mathrm{C}$ and $\mathrm{Cl}^{-} \ldots \mathrm{Cl}$ local stretching force constants used in main text to determine an approximate bond strength order.

Table S5: Vibrational frequencies for the $\mathrm{Cl}^{-} \ldots \mathrm{CCl}_{4}$ gas phase anion complex, featuring the halide at the face of the $\mathrm{CCl}_{4}$ molecule, from MP2/AVQZ calculations. Frequencies in $\mathrm{cm}^{-1}$, zero point energies (zpe) in $\mathrm{kJ} \mathrm{mol}^{-1}$, force constants in mDyne $\AA^{-1}$.

\begin{tabular}{cccc}
\hline Mode & Symmetry & Frequency & Force constant \\
\hline$\omega_{1}$ & $\mathrm{E}$ & 826 & 5.297 \\
$\omega_{2}$ & $\mathrm{E}$ & 826 & 5.297 \\
$\omega_{3}$ & $\mathrm{~A}_{1}$ & 753 & 4.394 \\
$\omega_{4}$ & $\mathrm{~A}_{1}$ & 475 & 4.584 \\
$\omega_{5}$ & $\mathrm{E}$ & 323 & 1.931 \\
$\omega_{6}$ & $\mathrm{E}$ & 323 & 1.931 \\
$\omega_{7}$ & $\mathrm{~A}_{1}$ & 313 & 1.867 \\
$\omega_{8}$ & $\mathrm{E}$ & 221 & 1.004 \\
$\omega_{9}$ & $\mathrm{E}$ & 221 & 1.004 \\
$\omega_{10}$ & $\mathrm{~A}_{1}$ & 77 & 0.117 \\
$\omega_{11}$ & $\mathrm{E}$ & 48 & 0.046 \\
$\omega_{12}$ & $\mathrm{E}$ & 48 & 0.046 \\
\hline zpe & 26.6 & & \\
\hline
\end{tabular}


Table S6: Vibrational frequencies for the $\mathrm{CCl}_{5}{ }^{-}$transition state from MP2/AVQZ calculations. Frequencies in $\mathrm{cm}^{-1}$, zero point energies (zpe) in $\mathrm{kJ} \mathrm{mol}^{-1}$, force constants in $\mathrm{mDyne} \AA^{-1}$.

\begin{tabular}{cccc}
\hline Mode & Symmetry & Frequency & Force constant \\
\hline$\omega_{1}$ & $\mathrm{E}^{\prime}$ & 919 & 6.562 \\
$\omega_{2}$ & $\mathrm{E}^{\prime}$ & 919 & 6.562 \\
$\omega_{3}$ & $\mathrm{~A}_{1}{ }^{\prime}$ & 460 & 4.365 \\
$\omega_{4}$ & $\mathrm{~A}_{2}^{\prime \prime}$ & 302 & 1.724 \\
$\omega_{5}$ & $\mathrm{E}^{\prime}$ & 289 & 1.535 \\
$\omega_{6}$ & $\mathrm{E}^{\prime}$ & 289 & 1.535 \\
$\omega_{7}$ & $\mathrm{E}^{\prime \prime}$ & 230 & 1.086 \\
$\omega_{8}$ & $\mathrm{E}^{\prime \prime}$ & 230 & 1.086 \\
$\omega_{9}$ & $\mathrm{E}^{\prime}$ & 134 & 0.363 \\
$\omega_{10}$ & $\mathrm{E}^{\prime}$ & 134 & 0.363 \\
$\omega_{11}$ & $\mathrm{~A}_{1}{ }^{\prime}$ & 123 & 0.314 \\
$\omega_{12}$ & $\mathrm{~A}_{2}{ }^{\prime \prime}$ & -557 & 2.367 \\
\hline zpe & 24.1 & & \\
\hline
\end{tabular}




\section{Cartesian Coordinates}

Table S7: Cartesian coordinates of the geometry of the halogen-appended $\mathrm{Cl}^{-} \ldots \mathrm{CCl}_{4}$ gas phase anion complex optimised at MP2/AVQZ, in $\AA$.

\begin{tabular}{ccccc}
\hline & & $\mathrm{x}$ & $\mathrm{y}$ & $\mathrm{z}$ \\
\hline & $\mathrm{C}$ & 0.000000 & 0.000000 & -0.817022 \\
$\mathrm{C}_{3 \mathrm{v}}$ & $\mathrm{Cl}$ & 0.000000 & 1.660488 & -1.452849 \\
& $\mathrm{Cl}$ & 0.000000 & 0.000000 & 0.933545 \\
& $\mathrm{Cl}$ & 1.438025 & -0.830244 & -1.452849 \\
& $\mathrm{Cl}$ & -1.438025 & -0.830244 & -1.452849 \\
$\mathrm{Cl}$ & 0.000000 & 0.000000 & 3.713364 \\
\hline
\end{tabular}

Table S8: Cartesian coordinates of the geometry of the $\mathrm{Cl}^{-} \ldots \mathrm{CCl}_{4}$ gas phase anion complex optimised at MP2/AVQZ, in $\AA$, featuring the halide at the face of the $\mathrm{CCl}_{4}$ molecule.

\begin{tabular}{ccccc}
\hline & & $\mathrm{x}$ & $\mathrm{y}$ & $\mathrm{z}$ \\
\hline & $\mathrm{C}$ & 0.000000 & 0.000000 & -0.668872 \\
$\mathrm{C}_{3 \mathrm{v}}$ & $\mathrm{Cl}$ & 0.000000 & 0.000000 & -2.461208 \\
& $\mathrm{Cl}$ & 0.000000 & 1.659347 & -0.113911 \\
& $\mathrm{Cl}$ & 1.437036 & -0.829673 & -0.113911 \\
& $\mathrm{Cl}$ & -1.437036 & -0.829673 & -0.113911 \\
& $\mathrm{Cl}$ & 0.000000 & 0.000000 & 3.039013 \\
\hline
\end{tabular}

Table S9: Cartesian coordinates of the geometry of the $\mathrm{CCl}_{5}{ }^{-}$transition state optimised at MP2/AVQZ, in $\AA$.

\begin{tabular}{|c|c|c|c|c|}
\hline & & $\mathrm{x}$ & $\mathrm{y}$ & $\mathrm{z}$ \\
\hline & $\mathrm{C}$ & 0.000000 & 0.000000 & 0.000000 \\
\hline & $\mathrm{Cl}$ & 0.000000 & 1.713893 & 0.000000 \\
\hline \multirow[t]{4}{*}{$\mathrm{D}_{3 \mathrm{~h}}$} & $\mathrm{Cl}$ & -1.484275 & -0.856946 & 0.000000 \\
\hline & $\mathrm{Cl}$ & 0.000000 & 0.000000 & 2.430953 \\
\hline & $\mathrm{Cl}$ & 1.484275 & -0.856946 & 0.000000 \\
\hline & $\mathrm{Cl}$ & 0.000000 & 0.000000 & -2.430953 \\
\hline
\end{tabular}

